\title{
Anderson localization in Euclidean random matrices
}

\author{
S. Ciliberti, ${ }^{1,2}$ T. S. Grigera, ${ }^{3}$ V. Martín-Mayor, ${ }^{1,2}$ G. Parisi, ${ }^{4}$ and P. Verrocchio ${ }^{1,2}$ \\ ${ }^{1}$ Departamento de Física Teórica I, Universidad Complutense de Madrid, Madrid 28040, Spain \\ ${ }^{2}$ Instituto de Biocomputación y Física de Sistemas Complejos (BIFI), Universidad de Zaragoza, 50009 Zaragoza, Spain \\ ${ }^{3}$ Instituto de Investigaciones Fisicoquímicas Teóricas y Aplicadas (INIFTA), c.c. 16, suc. 4, 1900 La Plata, Argentina \\ ${ }^{4}$ Dipartimento di Fisica, Università di Roma "La Sapienza," INFM unità di Roma I, \\ and Center for Statistical Mechanics and Complexity (SMC), Piazzale A. Moro 2, I-00185 Roma, Italy
}

(Received 16 December 2004; published 11 April 2005)

\begin{abstract}
We study the spectra and localization properties of Euclidean random matrices defined on a random graph. We introduce a powerful method to find the density of states and the localization threshold in topologically disordered (off-lattice) systems. We solve numerically an equation (exact on the random graph) for the probability distribution function of the diagonal elements of the resolvent matrix, with a population dynamics algorithm (PDA). We show that the localization threshold can be estimated by studying the stability of a population of real resolvents under the PDA. An application is given in the context of the instantaneous normal modes of a liquid.
\end{abstract}

DOI: 10.1103/PhysRevB.71.153104

PACS number(s): 61.43.Fs, 63.20.Pw

About forty years ago, Anderson ${ }^{1}$ showed that disorder can turn a system expected to be a metal from band theory into an insulator. Thus was born the difficult problem of Anderson localization. ${ }^{2-5}$ The physical picture is the following. In a pure system, states are described by Bloch wave functions. Disorder divides the energy band into localized regions (wave functions extending over a limited number of unit cells) and extended regions (wave functions are plane-wave-like, involving an extensive number of sites). The energy marking this division is called the mobility edge or localization threshold. If the Fermi level lies in the localized region, transport is strongly hampered. This problem has been much studied in electronic systems, where several numerical techniques combined with finite-size scaling allow precise determination of the mobility edge. ${ }^{6}$ Yet, few analytical calculations of a localization threshold are available. ${ }^{3,7}$ Given the large variety of systems described by random matrices, ${ }^{8}$ Anderson localization is significant beyond the physics of disordered metals. In this Brief Report we focus on the calculation of the mobility edge for Euclidean random matrices ${ }^{9}$ (ERMs), which we illustrate in a calculation of liquid instantaneous normal modes (INMs). ${ }^{10}$ The entries of a ERM are deterministic functions of (random) particle positions. These systems lack a reference lattice, so other approximation methods are needed, even for the calculation of the density of states. ${ }^{11,12}$ For the mobility edge, heuristic estimates (sometimes it is identified with the Ioffe-Regel limit or with the limits of the spectrum in some kind of effective-medium approximation ${ }^{13}$ ) or tentative numerical evaluations ${ }^{14}$ (hampered by the difficulty in reaching large system sizes) are used. Our scope is to estimate analytically the localization threshold for ERMs. Our approach is infinite dimensional, which is reasonable for liquids at high temperature (many-body correlations are negligible). We do not expect to estimate reliably quantities depending on the space dimension, such as critical exponents. However, above the lower critical dimension [expected to be 2 (Ref. 15)], the mean field should provide a reasonable estimate for the critical point, especially in con- densed systems with many interacting particles, as in our case. ERMs appear in the study of disordered $d$-wave superconductors, ${ }^{16}$ disordered magnetic semiconductors ${ }^{17}$ (very similar to a spin-glass model $^{18}$ ), INMs in liquids, ${ }^{11}$ vibrations in glasses, ${ }^{12}$ the gelation transition in polymers, ${ }^{19}$ and vibrations in DNA. ${ }^{20}$ Also, theoretical studies have been carried out. $^{21}$

Consider a translationally invariant system with potential energy $H[\vec{x}]=\sum_{i<j} v\left(\vec{x}_{i}-\vec{x}_{j}\right)$ [where $v(\vec{x})$ is the pair potential]. When dealing with high frequencies, ${ }^{10}$ it is enough to consider harmonic vibrations around the oscillation centers $\vec{x}^{c}$. We thus face the Hessian matrix $M_{i \mu, j \nu}\left[\vec{x}^{c}\right]$ $\equiv-v_{\mu \nu}\left(\vec{x}_{i}^{c}-\vec{x}_{j}^{c}\right)+\delta_{i j} \sum_{k=1}^{N} v_{\mu \nu}\left(\vec{x}_{i}^{c}-\vec{x}_{k}^{c}\right)$, where $v_{\mu \nu}(\vec{x})$ is the second derivative matrix of $v(\vec{x})$. Translational invariance (momentum conservation) implies that the sum of all elements in a row is zero. Once the probability density function (PDF) of the oscillation centers, $P\left[\vec{x}^{c}\right]$, is specified; the study of the spectral properties of the Hessian is a problem in ERM theory. ${ }^{9}$ In the context of supercooled liquids, three types of $P\left[\vec{x}^{c}\right]$ are usually considered: equilibrium configurations (INMs), ${ }^{10}$ minima (inherent structures), or stationary points of the potential energy. ${ }^{22}$ Here we restrict attention to localization properties of eigenvectors in the INM case with an emphasis on negative eigenvalues. The temperature evolution of this part of the spectrum is closely related to the dynamic slowing down close to the glass transition. ${ }^{10,14}$

We need to consider the resolvent matrix $^{2} R_{i \mu, j \nu}(z)$ $=(z-M)_{i \mu, j \nu}^{-1}(z$ is complex $)$. To study eigenvalues clustered around $\lambda$, we will set $z=\lambda+i \varepsilon$ for small $\varepsilon$. Our goal is to find an equation for the PDF of the diagonal term of the resolvent matrix $\mathcal{P}\left[\mathbf{R}_{i i} \mid z\right]$, focusing on its imaginary part [boldface symbols represent $3 \times 3$ matrices corresponding to particle displacements in three dimensions (3D)]. Consider the representation of $\mathbf{R}_{i i}(z)$ in terms of the eigenvectors $|\alpha\rangle$ of the matrix $M$ : 

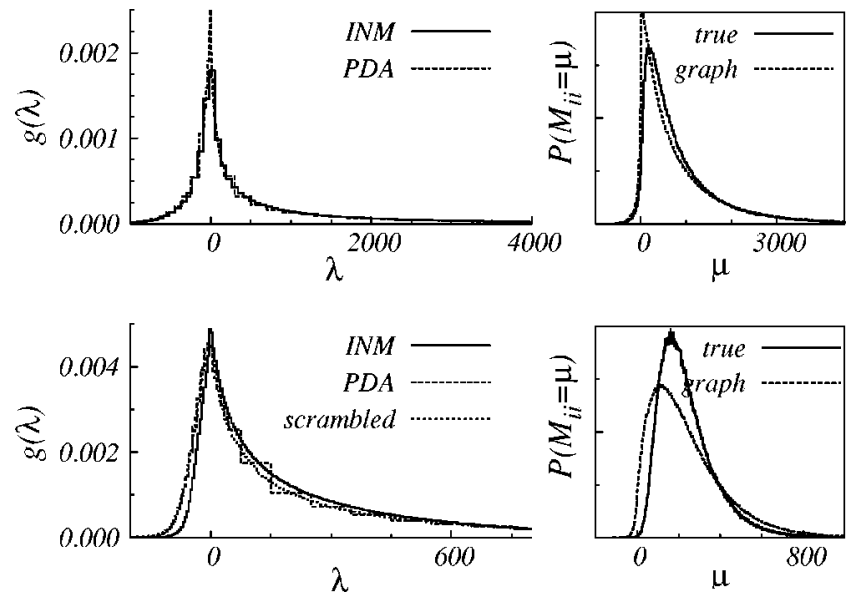

FIG. 1. Top. Left panel: DOS of a soft-sphere system at $T=7.72$ from Eq. (3) with $\varepsilon=1.0(N=20000)$ and from numerical diagonalization of the Hessian (100 configurations). Right panel: distribution of the diagonal terms of the Hessian, from Eq. (2) and from liquid simulations at $T=7.72$. Bottom. The same for $T=0.683$. In addition, we show the spectrum of the corresponding scrambled matrix. The clear difference in the distribution of the matrix elements is responsible for the deviations observed in the DOS at the lower temperature.

$$
\operatorname{Im} R_{j j}(\lambda+i \varepsilon)=\operatorname{Im} \sum_{\alpha} \frac{|\langle j \mid \alpha\rangle|^{2}}{\lambda+i \varepsilon-\lambda_{\alpha}} .
$$

The probability distribution $\mathcal{P}\left[\mathbf{R}_{i i} \mid z\right]$ will be rather peculiar in the localized region. The amplitude of eigenvectors $|\alpha\rangle$ with $\left|\lambda_{\alpha}-\lambda\right| \lesssim \varepsilon$ will be large for the main particle of the eigenmode, and will decrease exponentially with increasing distance. Let $n(\lambda)$ be the typical number of particles for which the amplitude of an eigenmode is sizable. The probability that particle $j$ significantly participates in an eigenvector of energy $\lambda_{\alpha} \in(\lambda-\varepsilon, \lambda+\varepsilon)$ is of order $\varepsilon g(\lambda) n(\lambda)[g(\lambda)$ is the density of states]. In this case $\operatorname{Im} R_{j j}$ is of order $1 /[\varepsilon n(\lambda)]$, while it is of order $\varepsilon$ with probability $1-\varepsilon g(\lambda) n(\lambda)$. Thus, the mean value of $\operatorname{Im} R_{j j}$ is finite for small $\varepsilon$ and proportional to $g(\lambda)$, but its variance is $\sigma^{2}(\lambda ; \varepsilon) \simeq g(\lambda) /[\varepsilon n(\lambda)]$ and diverges when $\varepsilon \rightarrow 0$. A more refined analysis ${ }^{3}$ shows that in the limit $\epsilon \rightarrow 0$ the PDF for $\operatorname{Im} R_{j j}$ decays as $\left(\operatorname{Im} R_{j j}\right)^{-\beta}, \beta \leqslant 1.5$, while at small but nonzero $\epsilon$ there is a cutoff that makes it strictly zero for $\operatorname{Im} R_{j j}>1 / \varepsilon$. On the other hand, in the extended region all the $|\langle j \mid \alpha\rangle|^{2}$ are $O(1 / N)$ and one can replace the sum with an integral. It follows that the typical value of $\operatorname{Im} R_{j j}$ is of order 1 when $\varepsilon=0^{+}$.

To get an equation for $\mathcal{P}\left[\mathbf{R}_{i i} \mid z\right]$ we have generalized the Cizeau-Bouchaud recursion relation ${ }^{7}$ to the case of 3D translationally invariant systems. This equation relates $\mathbf{R}_{i i}(z)$ in a system with $N$ particles to the full resolvent of a system where particle $i$ is not present anymore, but the positions of all other $N-1$ particles are as in the original system. The equation is obtained under two hypotheses: (i) that in the large- $N$ limit the off-diagonal matrix elements corresponding to two neighbors of the particle that is being removed can be neglected after removal; (ii) that three-point and higher cor-
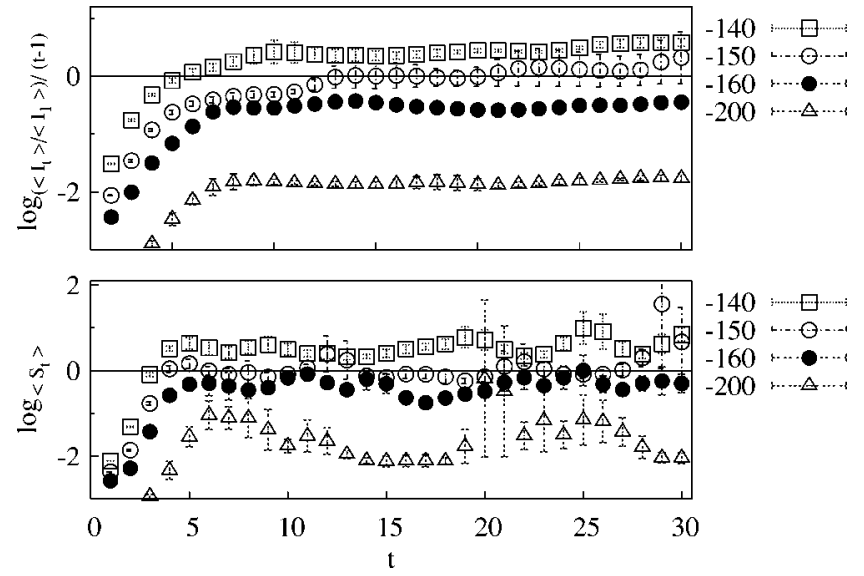

FIG. 2. Evolution of the median (top) and geometrical average (bottom) of the population of imaginary parts of the resolvent (see text) for $\lambda=-140,-150,-160$, and -200 . Data for $N=10^{6}$.

relations can be ignored, i.e., the off-diagonal matrix elements $M_{i \mu, j \nu}\left[\mathbf{x}^{c}\right]$ are treated as independent random variables, $\mathbf{P}[\mathbf{M}]=\Pi_{i<j} \mathrm{p}\left(\mathbf{M}_{i j}\right)$ (diagonal elements are fixed by translational invariance). The PDF of the single matrix element is then

$$
\mathrm{p}\left(\mathbf{M}_{i j}\right)=\left(1-\frac{\gamma}{N}\right) \delta\left(\mathbf{M}_{i j}\right)+\frac{\rho}{N} \int_{0}^{r_{\mathrm{c}}} d r g^{(2)}(r) \delta\left(\mathbf{M}_{i j}+\mathbf{v}^{\prime \prime}(\vec{r})\right),
$$

where $g^{(2)}(r)$ is the radial distribution function and $\gamma \equiv 4 \pi \rho \int_{0}^{r_{\mathrm{c}}} d r^{\prime} r^{\prime 2} g^{(2)}\left(r^{\prime}\right)$ is the average number of particles whose distance from particle $i$ is less than the cutoff $r_{c}$ (in our case $\gamma \approx 32$; thus the probability of finding disconnected clusters is negligible ${ }^{23}$ ). Under these hypotheses the following equation holds ${ }^{24}$ for the resolvent of the infinite system (note that the inverse-matrix symbols refer to the $3 \times 3$ matrices and that the $\mathbf{R}_{j j}^{-1}$ are independently chosen from the $\mathcal{P}\left[\mathbf{R}_{j j} \mid z\right]$ distribution):

$$
\begin{aligned}
\mathcal{P}\left[\mathbf{R}_{i i} \mid z\right]= & \int d \mathrm{P}[\mathbf{M}] d \mathcal{P}\left[\mathbf{R}_{j j} \mid z\right] \delta\left[\mathbf{R}_{i i}-\left(z+\sum_{j \neq i} \mathbf{M}_{i, j}\right.\right. \\
& \left.\left.-\sum_{j \neq i} \mathbf{M}_{i j}\left(\mathbf{R}_{j j}^{-1}+\mathbf{M}_{i j}\right)^{-1} \mathbf{M}_{j i}\right)^{-1}\right] .
\end{aligned}
$$

Equation (3) becomes exact in the infinite-dimensional random graph case ${ }^{23}$ where each particle is the root of a Cayley tree whose fluctuating connectivity is distributed with a Poisson law of mean value $\gamma$.

We consider the INM of a monoatomic system with potential (in natural units) $v(r)=(1 / r)^{12}$ (see Grigera et al. in Ref. 22 for details) and $\rho=1$. We obtained the pair correlation function by means of a Monte Carlo simulation of an $N=2048$ system, for temperatures $T$ between 0.683 and 123 , in the liquid phase (crystallization happens at $T_{c} \approx 0.592$ ). Once $g^{(2)}\left(r^{\prime}\right)$ is known, one needs to solve Eq. (3). We have done this numerically using a population dynamics algorithm. Starting from a population of $N$ elements with $\mathbf{R}_{i i}(z ; t=0)=\mathbf{I} / z$, we iterate the scheme 


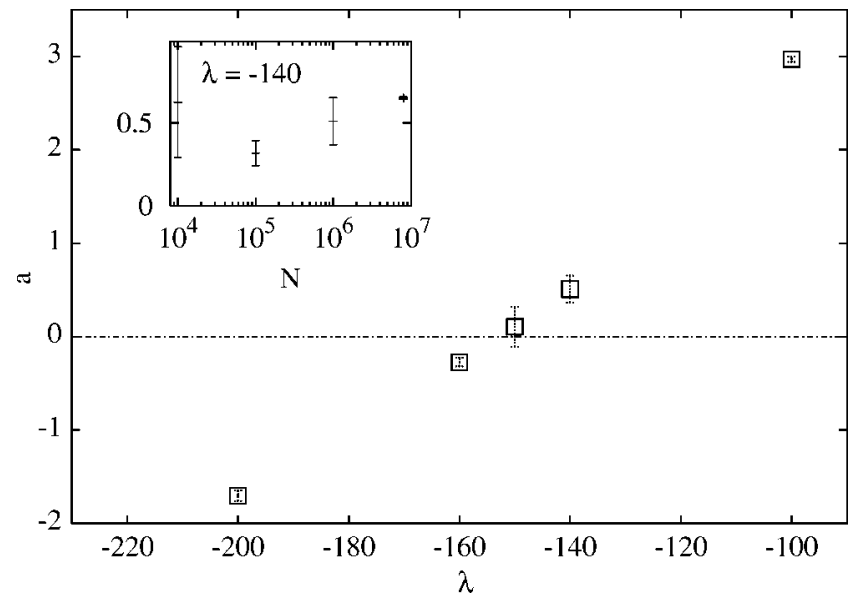

FIG. 3. Values of the slope from a linear fit of $\left\langle\ln I_{t}\right\rangle$ for $t>10$. The mobility edge is estimated at $\lambda=-152(5)$. Inset: $N$ dependence of the slope.

$$
\begin{gathered}
\mathbf{R}_{i i}(z ; t+1)=\mathbf{F}\left(\mathbf{R}_{j j}(z ; t), \mathbf{M}_{i j}\right) \\
\mathbf{F}\left(\mathbf{R}_{j j}, \mathbf{M}_{i j}\right) \equiv\left[z \mathbf{I}+\sum_{j} \mathbf{M}_{i j}-\sum_{j \neq i} \mathbf{M}_{i j}\left(\mathbf{R}_{j j}^{-1}+\mathbf{M}_{i j}\right)^{-1} \mathbf{M}_{j i}\right]^{-1} .
\end{gathered}
$$

To form the sums in Eq. (5), we divide the space around particle $i$ up to the cutoff distance, into spherical shells of width $\Delta r=r^{c} / 4096$. The probability of having a particle in the shell is $4 \pi \rho \int_{r}^{r+\Delta r} d r^{\prime} r^{\prime 2} g^{(2)}\left(r^{\prime}\right)$. Both the identity $j$ of the particle interacting with $i$ and the direction of the vector $\vec{r} \equiv \vec{r}_{i}-\vec{r}_{j}$ are chosen randomly with uniform probability. One step consists in the sequential update of the full population. After 100 steps the first moments of the probability distribution are constant, apart from fluctuations due to the finite value of $N$. To decrease them, we used (typically) 300 more time steps. The population averages of $\operatorname{Im} R_{i \mu, i \mu}$ and $\left(\operatorname{Im} R_{i \mu, i \mu}\right)^{2}$ are calculated, then time averaged. From them we compute the density of states (DOS), $g(\lambda)=-\left\langle\operatorname{Im} R_{i \mu, i \mu}\right\rangle / \pi$, and $\left\langle\operatorname{Im} R_{i \mu, i \mu}^{2}\right\rangle$.

We have computed the DOS both from Eq. (3) and by numerical diagonalization of the Hessian. As Fig. 1 (left) shows, agreement is good at high temperature, where threebody correlations are less important. The mild disagreement at $T=0.683$ is due to neglected high-order correlations. Indeed, the outcome of Eq. (3) is identical to the DOS of the scrambled Hessian $\tilde{\mathbf{M}}$ (Fig. 1, lower left), which is built by picking its off-diagonal elements randomly and without repetition from the off-diagonal elements of the true Hessian, and then imposing symmetry and translational invariance. This destroys three-body and higher correlations. Neglected correlations are responsible for the low-temperature discrepancies between Eq. (3) and numerical digonalization. Indeed, compare the PDF of the diagonal term of the Hessian matrix as computed from Eq. (2) and from the actual liquid configurations (Fig. 1, right). Since this PDF coincides with the DOS at leading order in perturbation theory (the diagonal term is much larger than the typical off-diagonal term), we

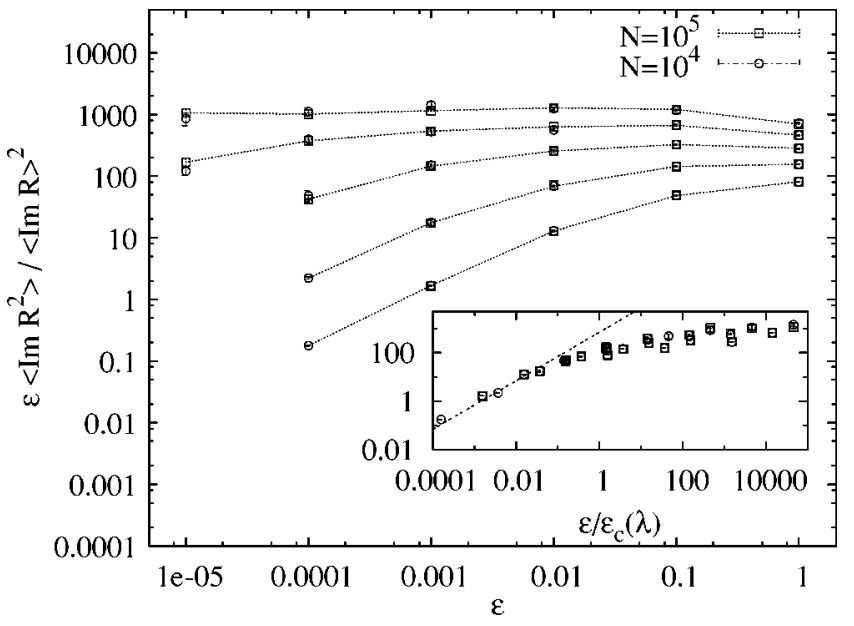

FIG. 4. $l(\lambda ; \varepsilon)$ vs $\varepsilon$ near the lower mobility edge at $T=0.683$ for (from top to bottom) $\lambda=-140,-130,-120,-110$, and -100 . Inset: Scaling plot $l(\lambda ; \varepsilon)$ vs $\varepsilon / \varepsilon_{c}(\lambda)$ for $\lambda \geqslant-130$.

understand why at $T=0.683 \mathrm{Eq}$. (3) overestimates the weight of the imaginary frequencies.

The main result of our work is the introduction of a way to determine the localization threshold which generalizes the method of Abou-Chacra et $a .^{3}$ to the case of topologically disordered systems (i.e., those described by ERMs). Since in the localized region and in the $\varepsilon \rightarrow 0$ limit, $\operatorname{Im} \mathbf{R}_{i i}=0$ except for a vanishing fraction of particles, a population of purely real resolvents must be stable under Eq. (5). The idea ${ }^{3}$ is to start with an equilibrated population of real resolvents (setting $z=\lambda$ ), add a small imaginary part, and evolve the population to see whether the imaginary parts grow (extended phase) or disappear (localized phase).

We have analyzed in this way the negative mobility edge at $T=0.683$. We added the imaginary part to a population of equilibrated real resolvents, and let it evolve with the linearized (with respect to the imaginary part) form of Eq. (5). We evolved the real part with Eq. (5) as if the population were real. Since the PDF of $\operatorname{Im} \mathbf{R}_{j j}^{(t)}$ is extremely broad, one must choose carefully the quantity to examine. In Ref. 3 it was proposed to consider the quotient $S_{t}$ of the geometric mean of the imaginary parts at times $t$ and $t-1 . S_{t}$ was assumed to be $t$ independent, and it was expected to be equal to 1 at the mobility edge. Unfortunately, due to the presence of poles at zero imaginary part in the recursion equation, the average of the imaginary parts at time $t$ is divergent. If we ignore this divergence we find (Fig. 2) that this quantity has extremely large fluctuations that do not vanish for large $N$, and is useless to determine the threshold. Fortunately, the median $I_{t}$ of the PDF of $\operatorname{Im} \mathbf{R}_{i i}^{(t)}$ does not have these drawbacks and is much more reliable. However, obtaining asymptotic estimates for $I_{t}$ requires values of $N$ that grow fast with $t$. For $t<15$ we have found no differences between $N=10^{6}$ and $N=8 \times 10^{6}$. One would expect that $\ln I_{t} \sim a t$, with $a$ less than (greater than) zero in the localized (extended) phase, and this is indeed what we find at large $t$ (Fig. 2). For $N=10^{6}$ we utilized ten samples, estimating the errors via the jackknife method. A linear fit of $\ln I_{t}$, for $1 \leqslant t \leqslant 15$ gives values $a$ that locate the mobility edge at $\lambda=-152(5)$ (Fig. 3). 
A similar analysis gives, as the upper limit of the extended region, $\lambda=1611(80)$.

We also considered a way to locate the threshold through the behavior of the variance of $\operatorname{Im} R$. Consider $l(\lambda ; \varepsilon)$ $\equiv \varepsilon\left\langle\operatorname{Im} R^{2}\right\rangle /\langle\operatorname{Im} R\rangle^{2}$. From the remarks above it follows that in the localized region and for small $\varepsilon, l(\lambda ; \varepsilon)$ tends to a constant, while in the extended region the variance is finite and $l(\lambda ; \varepsilon)$ is $O(\varepsilon)$. Unfortunately this approach has a major limitation: for the higher values of $\lambda$, the extended, $O(\varepsilon)$ regime is reached, but as the threshold is approached the regime sets in for lower and lower values of $\varepsilon$, making the threshold very difficult to estimate. To overcome this problem we have tried the phenomenological scaling $l(\lambda, \varepsilon)=\varepsilon_{c}^{\alpha} f\left(\varepsilon / \varepsilon_{c}(\lambda)\right)$, with $\alpha=0, f(x) \sim x$ for $x \sim 0$, and $\varepsilon_{c}(\lambda) \rightarrow 0$ for $\lambda \rightarrow \lambda_{\text {th }}$ (Fig. 4). The $\varepsilon_{c}(\lambda)$ (that we obtained only in the range $\lambda \geqslant-130$ ) can be fitted to a power law $c(\lambda-\lambda)^{\gamma}$, with $\gamma=21$ and $\lambda_{\text {th }}=-171.6$. The fit is not reliable, since it does not reach close enough to the critical point. ${ }^{25}$ This huge exponent is perhaps an indication of an essential singularity at the mobility edge (cf. the results of Ref. 5) or of a wrong choice of $\alpha$ (which cannot be determined reliably).
In summary, we have studied Anderson localization in Euclidean random matrices. Disregarding three-particle and higher correlations (exact for an infinite-dimensional system), an equation was found for the PDF of the diagonal element of the resolvent matrix, which was solved numerically with a population dynamics algorithm. This approximation works well at high temperature, but significantly overestimates the weight of negative modes close to crystallization. The mobility edge can be located by studying the stability of a population of real resolvents, something we have done numerically, obtaining reliable values for the threshold in topologically disordered systems.

We acknowledge partial support from MCyT, Spain (Grants No. FPA2001-1813, No. FPA2000-0956, and No. BFM2003-08532-C03) and ANPCyT, Argentina. S.C. was supported by the ECHP program (Grant No. HPRN-CT2002-00307). V.M.-M. was supported by the Ramón y Cajal program, and P.V. by the European Commission (Grant No. MCFI-2002-01262). T.S.G. was supported by CONICET (Argentina).
${ }^{1}$ P. W. Anderson, Phys. Rev. 109, 1492 (1958).

${ }^{2}$ J. T. Edwards and D. J. Thouless, J. Phys. C 5, 807 (1972); D. J. Thouless, Phys. Rep., Phys. Lett. 13, 93 (1974).

${ }^{3}$ R. Abou-Chacra, P. W. Anderson, and D. J. Thouless, J. Phys. C 6, 1734 (1973).

${ }^{4}$ P. A. Lee and T. V. Ramakrishnan, Rev. Mod. Phys. 57, 287 (1985).

${ }^{5}$ Y. V. Fyodorov, A. D. Mirlin, and H.-J. Sommers, J. Phys. I 2, 1571 (1992).

${ }^{6}$ See Localisation 2002 [J. Phys. Soc. Jpn. 72A (2003)].

${ }^{7}$ P. Cizeau and J. P. Bouchaud, Phys. Rev. E 50, 1810 (1994).

${ }^{8}$ M. L. Mehta, Random Matrices (Academic Press, New York, 1991).

${ }^{9}$ M. Mézard, G. Parisi, and A. Zee, Nucl. Phys. B 559, 689 (1999).

${ }^{10}$ T. Keyes, J. Chem. Phys. 101, 5081 (1994).

${ }^{11}$ T. M. Wu and R. F. Loring, J. Chem. Phys. 97, 8568 (1992); Y. Wan and R. Stratt, ibid. 100, 5123 (1994); A. Cavagna, I. Giardina, and G. Parisi, Phys. Rev. Lett. 83, 108 (1999); J. Phys.: Condens. Matter 12, 6295 (2000).

${ }^{12}$ S. Ciliberti, T. S. Grigera, V. Martin-Mayor, G. Parisi, and P. Verrocchio, J. Chem. Phys. 119, 8577 (2003); T. S. Grigera, V. Martin-Mayor, G. Parisi, and P. Verrocchio, Phys. Rev. Lett. 87, 085502 (2001).

${ }^{13}$ G. Biroli and R. Monasson, J. Phys. A 32, L255 (1999); R. Monasson, Eur. Phys. J. B 12, 555 (1999).

${ }^{14}$ J. Fabian, Phys. Rev. B 55, R3328 (1997); S. D. Bembenek, and B. Laird, Phys. Rev. Lett. 74, 936 (1995); S. Ciliberti and T. S.
Grigera, Phys. Rev. E 70, 061502 (2004).

${ }^{15}$ P. W. Anderson, D. J. Thouless, E. Abrahams, and D. S. Fisher, Phys. Rev. B 22, 3519 (1980).

${ }^{16}$ C. Chamon and C. Mudry, Phys. Rev. B 63, 100503(R) (2001).

${ }^{17}$ L. Brey and G. Gómez-Santos, Phys. Rev. B 68, 115206 (2003).

${ }^{18}$ D. S. Dean and D. Lancaster, Phys. Rev. Lett. 77, 3037 (1996).

${ }^{19}$ K. Broderix, T. Aspelmaier, A. K. Hartmann, and A. Zippelius, Phys. Rev. E 64, 021404 (2001).

${ }^{20}$ S. Cocco and R. Monasson, Phys. Rev. Lett. 83, 5178 (1999).

${ }^{21}$ E. Bogomonly, O. Bohigas, and C. Schmit, J. Phys. A 36, 3595 (2003); C. R. Offer and B. D. Simons, ibid. 33, 7567 (2000); A. Zee and I. Affleck, J. Phys.: Condens. Matter 12, 8863 (2000); D. S. Dean, J. Phys. A 35, L153 (2002).

${ }^{22}$ F. H. Stillinger and T. A. Weber, Science 225, 983 (1984); L. Angelani, R. Di Leonardo, G. Ruocco, A. Scala, and F. Sciortino, Phys. Rev. Lett. 85, 5356 (2000); K. Broderix, K. K. Bhattacharya, A. Cavagna, A. Zippelius, and I. Giardina, ibid. 85, 5360 (2000); T. S. Grigera, A. Cavagna, I. Giardina, and G. Parisi, ibid. 88, 055502 (2002); D. J. Wales and J. P. K. Doye, cond-mat/0309059(unpublished).

${ }^{23}$ A. J. Bray and G. J. Rodgers, Phys. Rev. B 38, 11461 (1988).

${ }^{24}$ S. Ciliberti, Ph.D. thesis, Università di Roma La Sapienza, 2003; S. Ciliberti, T. S. Grigera, V. Martin-Mayor, G. Parisi, and P. Verrocchio (unpublished).

${ }^{25}$ Moving nearer requires lowering $\varepsilon$, which is computationally expensive since the statistics must grow as $1 / \sqrt{\varepsilon}$ when the variance is growing as $1 / \varepsilon$. 